\title{
BMJ Open Quality Process improvement for a complex dual medical procedure
}

\author{
Wade Anthony Weigel, ${ }^{1}$ Michael Gluck, ${ }^{2}$ Andrew S Ross, ${ }^{2}$ Otto S Lin, ${ }^{2}$ \\ Barbara L Williams, ${ }^{3}$ Craig C Blackmore ${ }^{4}$
}

To cite: Weigel WA, Gluck M, Ross AS, et al. Process improvement for a complex dual medical procedure. BMJ Open Quality 2018:7:e000273. doi:10.1136/ bmjoq-2017-000273

Received 30 November 2017 Accepted 25 July 2018
Check for updates

(c) Author(s) (or their employer(s)) 2018. Re-use permitted under CC BY-NC. No commercial re-use. See rights and permissions. Published by BMJ.

${ }^{1}$ Department of Anesthesiology, Virginia Mason Medical Center, Seattle, Washington, USA

${ }^{2}$ Department of

Gastroenterology, Virginia

Mason Medical Center, Seattle, Washington, USA

${ }^{3}$ Center for Healthcare Improvement Science, Virginia Mason Medical Center, Seattle, Washington, USA

${ }^{4}$ Department of Radiology, Virginia Mason Medical Center, Seattle, Washington, USA

Correspondence to Dr Wade Anthony Weigel; wade.weigel@virginiamason.org

\section{ABSTRACT}

Pancreatic extracorporeal shock wave lithotripsy followed by endoscopic retrograde cholangiopancreatography is accepted worldwide as a treatment for a large, symptomatic, obstructing pancreatic stones. However, timely completion of the combined process requires coordination of equipment and personnel from two different complex procedures.

We used Lean management tools in a week-long event to redesign the process around the patient. Using ideagenerated Plan Do Study Act cycles to refine the process, from scheduling to postprocedure recovery, equipment and personnel were aligned to allow these two procedures to occur in immediate succession.

The redesigned process resulted in all patients receiving both procedures without delay. This eliminated over 8 hours of wait time. Standard work and a newly created complex scheduler improved flow. We reduced the number of anaesthetics for patients without prolonging the procedure length.

\section{PROBLEM}

For combined extracorporeal shock wave lithotripsy (ESWL) / endoscopic retrograde cholangiopancreatography (ERCP) procedures, time delay from the completion of the ESWL to the ERCP has been shown to be detrimental to patient outcomes. Delaying the ERCP for 24 hours after the ESWL can increase the pain medication requirements for patients. In our institution, for these combined procedures, the ERCP was commonly performed later the same day or, at times, delayed to another day.

The goal of this project was to create the perfect patient experience by simplifying, streamlining and mistake-proofing scheduling and day of procedure flow for the combined ESWL/ERCP procedure. We sought to minimise the time between procedures to limit the risk of complications. Anaesthesia was administered to patients undergoing the ESWL and the ERCP. There are three phases to the administration of an anaesthetic (induction, maintenance and emergence) of which maintenance infers the lowest potential for adverse anaesthetic events. By 3 months after the process improvement event, the goal was to have the infrastructure in place to ensure that all ESWL/ERCP cases were scheduled and performed in immediate succession at one location with the administration of only one anaesthetic.

Patient safety alerts (PSAs) are used in our hospital to call attention to patient care improvement opportunities. This system provided documentation about problems associated with separating the ESWL and ERCP in time and space. Multiple ESWL/ ERCP-related PSAs reported increased risk to patients from being anaesthetised twice when one should suffice, documented multiple procedural cancellations due to confusing preprocedure instructions on aspirin and described delays in the ESWL because of no ERCP consent. The presence of these PSAs raised awareness that change was needed.

For analysis of this quality improvement project, a waiver was granted from our Institutional Review Board. The BMJ Open Quality template was used in preparation of this manuscript. This work had already been partially presented as an abstract. ${ }^{2}$ This project occurred in the context of a hospital system committed to process improvements using the Lean methodology of Virginia Mason Production System (VMPS). ${ }^{3}$ VMPS was introduced in 2000 and has been used for process improvements throughout the hospital system. Virginia Mason is a 300-bed urban hospital with 28 anaesthetising locations (24 operating rooms (ORs) and 4 out of OR locations).

\section{BACKGROUND}

Pancreatic stones, a complication of chronic pancreatitis, can cause debilitating pain resulting in the use of high doses of narcotics, admissions to the hospital, inability to work and diminished quality of life. Surgery is a treatment option for symptomatic pancreatic stones, but this has been associated with mixed results, high cost and significant complications such as pancreatic duct leaks, bleeding and large incisional hernias. Endoscopic approaches, including ERCP 
with pancreatic sphincterotomy and mechanical stone extraction, have been used with some success; however, stones that are lodged behind main duct strictures and those over $5 \mathrm{~mm}$ are exceedingly difficult to remove. To facilitate endoscopic removal, pancreatic ESWL has been enlisted to fragment stones resulting in spontaneous passage of some stones and reduction in size of others so that endoscopically delivered baskets and balloons can be used to extract stone segments. ${ }^{4}$ Studies have shown that combining ESWL with ERCP for stone extraction decreased pain, hospitalisations, need for surgery and narcotic pain medications, as well as improved stone removal success rate and quality of life. ${ }^{156}$ This technique is considered around the world to be the mainstay of care for large pancreatic stone removal. ${ }^{789}$

Only a few medical centres in the USA have the capability of combining ESWL and ERCP. Originally, the process at our hospital was designed around the equipment. Lithotripsy, before 2008, was performed with a stationary lithotripter in a designated OR (Dornier HM3 Lithotripter, Donier, Dornier Medtech, Munich, Germany). Using this lithotripter unit in combination with the need for high-quality imaging for ERCP (located in the Gastrointestinal procedural rooms) necessitated different sites for the two phases ESWL/ERCP procedure. The stationary lithotripter unit was replaced by a mobile unit in 2008 (Storz Medical Modulith SLX-F2, Karl Storz Lithotripsy America, Kennesaw, Georgia, USA). Reports on mobile lithotripter pancreatic ESWL at our institution and others have demonstrated very good results. ${ }^{1610}$ With the mobile lithotripter came the opportunity to design this process around the patient, a philosophy embraced by our institution.

This project was conducted outside the OR, in a venue for anaesthesiologists known as non-operating room anaesthesia (NORA). In recent years, there has been an increase in demand for anaesthesia outside the OR to care for sicker patients undergoing increasingly complex procedures. Complicating this situation is the unfamiliarity and lack of resources outside the OR for the provision of high-quality anaesthesia care. At our institution, in recent years, we have seen double-digit increases in NORA cases, and we have responded with aggressive efforts to provide appropriate equipment and personnel to these areas.

Scheduling a combined ESWL/ERCP procedure involved coordination of three services (GI, urology and anaesthesiology), in two sites (OR and procedural suite). Our schedulers struggled to coordinate these variables with patient availability that led to inefficiencies. It has been shown that procedural scheduling delays dissatisfy patients in GI procedural units. ${ }^{11} 12$ Therefore, we also set out to improve scheduling of this procedure.

A barrier to complete this work was the need to retrofit a room to accommodate all of the required equipment. More daunting, however, was coordinating three services (urology, gastroenterology and anaesthesiology). Gains in efficiency and patient satisfaction have been reported in the gastroenterology procedural setting using Lean methods. ${ }^{13}$ Because of the strong central governance built around Lean process improvement at our hospital, we had the support of hospital administration and expected this project to be successful. ${ }^{14}$

\section{MEASUREMENT}

We reviewed our hospital PSAs that included the terms ERCP and ESWL. We also cross-reference the PSA database with our list of all patients undergoing ESWL/ERCP from 2011 to 2017. A reduction in PSAs would suggest improved patient safety. We were able to capture some of the most important measurements for patient safety such as the number of anaesthetics administered per visit, the number of cases completed within 24 hours and total procedure time. Gains in patient safety would be reflected by a reduction in the number of anaesthetics administered to one patient, more dual procedures completed within 24 hours and no change in total procedure time. Procedure times, before the intervention, combined the time for the ESWL and ERCP individually. If the intervention was done correctly, then the equipment and personnel should be present afterwards to allow the same efficiency for completion of the ESWL/ERCP procedure. To capture cost implications, we evaluated patient length of stay, standard anaesthetic charge generation and lithotripter repairs. Implications for the urologists and gastroenterologists productivity were evaluated by physician feedback.

We received a list of all hospital inpatient and observational patients from our billing department with a pancreatitis diagnosis (International classification of disease (ICD) $9=577$ ) and ESWL procedure (ICD9=98.59 or 98.52) between January 2011 and March 2013. We received a list of patients from the procedural unit electronic record for May 2013 to June 2017 identifying patients who had undergone the ESW/ERCP procedure. Patient demographics were received from billing. One author (WW) reviewed all the patient medical records to confirm the performance of these two procedures and to collect admission dates, procedural anaesthesia times and discharge dates.

We used intent-to-treat method in our analysis, in which all procedures prior to the intervention date (May 2013) were considered preintervention even though, on occasion, the two procedures may have been performed on the same day. T-tests were used to analyse continuous variables, and crosstabs were used to analyse dichotomous variables. All analyses were performed using StataMP V.12.

\section{DESIGN}

A multidisciplinary team undertook a 1 week rapid process improvement work project in April 2013. The team was composed of an executive sponsor (senior vice president), an executive workshop leader (vice president) and a Virginia Mason Production System specialist 
as the team leader. Team members included an anaesthesiologist, urologist, gastroenterologist, section manager, surgical scheduler, outside hospital representative and an internal medicine resident. We defined the process as beginning with first contact (by phone, outpatient visit or day of procedure consultation) in the GI department and ending with discharge from the GI procedural unit. Preliminary work included interviews of involved personnel as well as a compilation of all work done by schedulers, staff and managers in each of the respective sections. We observed and interviewed day of procedure providers and staff as well as patients and families. Flow maps were created with notations about opportunities to improve specific segments of the operations.

This effort led us to identify several specific areas where improvements were needed in the combined ESWL/ ERCP procedure. First and foremost, there was a need for coordination between the gastroenterology, urology and anaesthesiology departments for efficient scheduling. Patient and family issues included separate and conflicting preprocedural information packets from urology and gastroenterology and day of procedure confusion regarding site of check-in, site of recovery and family waiting. Other day of procedure problems included the need for administration of two separate anaesthetics, recovery nursing order confusion between procedures and the delay between procedures.

Accordingly, we designed the work of the process improvement team around three themes:

- Theme 1: improving scheduling.

- Theme 2: reducing patient and family confusion.

- Theme 3: eliminating procedure delays.

\section{STRATEGY}

The process improvement team engaged in quality improvement activities specific to each theme over the course of the event.

\section{Theme 1: improving scheduling}

We recognised that patient access was compromised by the complex task of aligning two procedures, ESWL and ERCP, that were not otherwise coordinated and for which there was no standard work. The urology and gastroenterology scheduling departments were working in silos with little resources available to facilitate a joint procedure. Plan Do Study Act (PDSA) cycles were conducted in order to improve the scheduling process. Through these improvement cycles, a process for scheduling this complex procedure was created, which included a designated complex scheduler and standard work that facilitated communication between the GI and urology schedulers. The use of a complex scheduler has been used to improve scheduling efficiency for complex GI procedures elsewhere. ${ }^{15}$ These changes led to more clarity for the schedulers, fewer interoffice phone calls and ultimately a simplified and streamlined scheduling process. Success was determined informally by the schedulers and the process improvement team's assessment of usability.
We initially implemented the improved scheduling with limited block times. An ESWL/ERCP could only be scheduled 1 day per week, which was different for each week of the month. We imposed these restrictions to accommodate the clinical duties, administrative meetings and educational commitments for the departments of gastroenterology, urology and anaesthesiology. However, soon after implementing the new scheduling process, we realised case volumes were higher than anticipated. We responded by readdressing each department's time constraints, which led to multiple compromises, and ultimately, the current state of daily ESWL/ERCP block time was created.

\section{Theme 2: reducing patient and family confusion}

We analysed the patient and family experience and realised there was conflicting information in the procedural information packets sent out separately by each department. Of note, the restriction of non-steroidal anti-inflammatory drugs (NSAIDs) by the urology department, but not the GI department, led to patient confusion. Patients would follow the GI instructions, which allowed NSAID ingestion, only to be told they were non-compliant with the urology requirement to avoid NSAIDs and have their case cancelled. This problem was documented multiple times in our PSA system. We facilitated the creation of a single ESWL/ERCP information packet in conjunction with and approved by both departments.

Another patient family experience issue was the logistics on the day of the procedure. Confusion was perpetuated by virtue of two related yet functionally uncoupled procedures being performed on the same day. The morning of the ESWL, the patient would be asked at the check-in desk, 'Will you be receiving an ERCP with your ESWL today?'. The patient checked in at the outpatient surgery desk in the first building and walked to the ESWL preprocedural prep area in a second building. Then, the patient would undergo the ESWL procedure and be transported to the surgical recovery area in the second building. After recovery, the patient was transported to the GI procedural area in a third building where, on ERCP completion, the patient was cared for in that procedural recovery area (figure 1). During the course of this movement spanning three buildings, the families tended to be unaware of the physical whereabouts of their loved ones and unsure where to wait to hear from the treating physician (urologist or gastroenterologist). The solution to this logistics puzzle was to have the patient check in to one area where all procedures, consultations and recovery would take place.

\section{Theme 3: eliminating procedure delays}

Proper procedural consent requires an uncompromised level of patient cognition. Therefore, both consents are needed to be completed before the first procedure. However, the ESWL would often be delayed due to lack of ERCP consent when the procedures were separated in time and place. Order writing was also problematic 

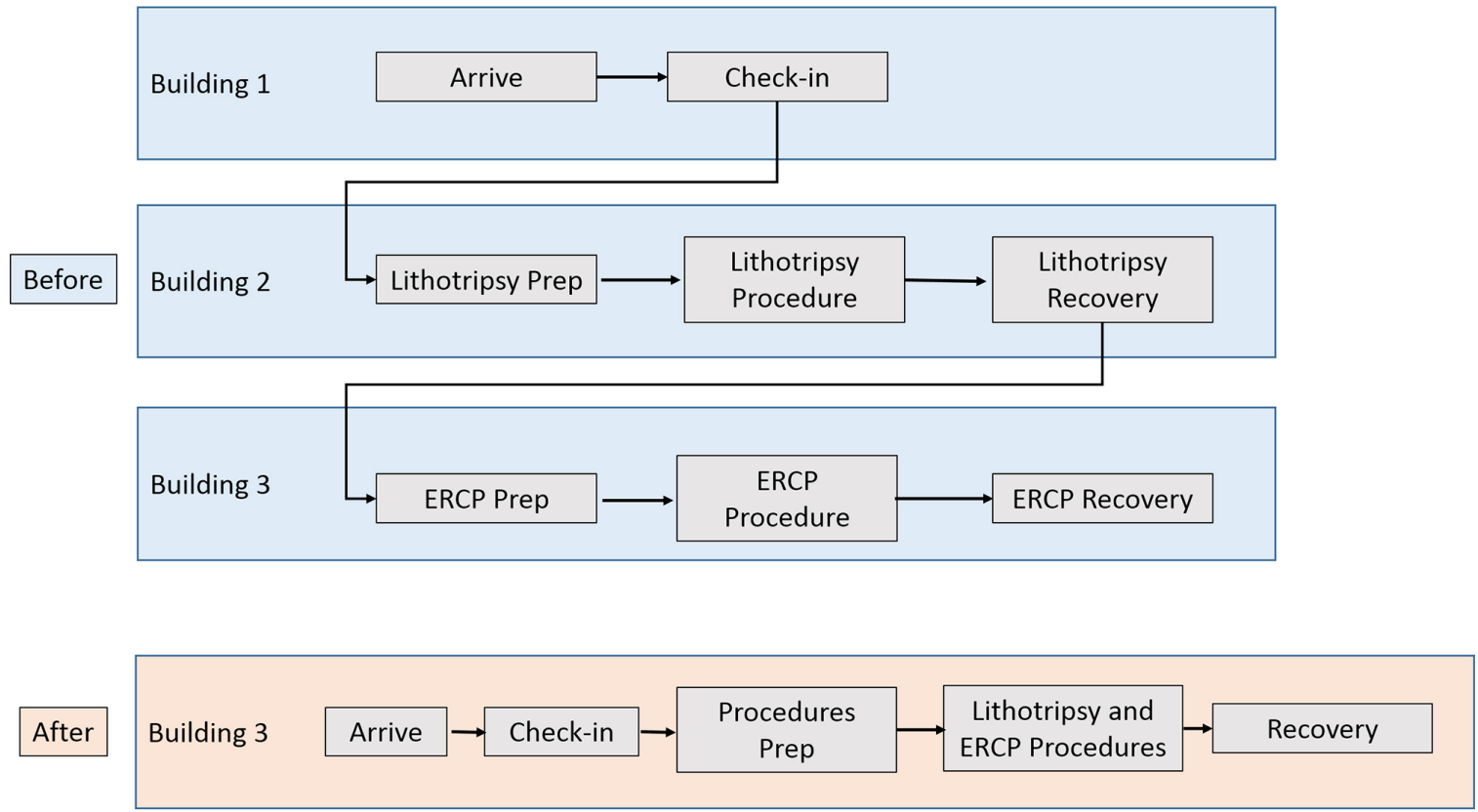

Figure 1 Swim lane diagram of patient and family movement before and after the intervention. ERCP, endoscopic retrograde cholangiopancreatography.

following the ESWL, as recovery nurses did not know which provider to contact since urology was a consult service and gastroenterology had yet to assume care for the patient. After the process improvement event, it became a requirement that both the urologist and gastroenterologist be present before the dual procedure to eliminate ESWL delays due to the lack of ERCP consent. Recovery orders were written by the gastroenterologist, per standard practice after an ERCP.

Time between procedure delays could most effectively be eliminated if both the ESWL and ERCP were completed in immediate succession in the same location. Given the obvious benefits of a single location approach for this procedure, the key question became which location, the OR or the procedural suite would be more appropriate. The team examined both locations and queried the procedural physicians about the pros and cons of each. Two important considerations were quality of imaging and proximity of supplies. For the urologist, there was little need for a supply of equipment besides the lithotripter. The imaging required for lithotripsy consisted of a portable c-arm to aim the beam. For the gastroenterologist, however, there was a very different set of requirements for imaging and equipment. They rely on high-quality imaging for pancreatic stone identification and stent placement. This high-quality fluoroscopy was only located in the gastroenterology suite and could not be transported. Gastroenterologists require multiple stents and balloons for pancreatic stone removal followed by pancreatic duct stent placement. This equipment is located in the procedural unit and would be time consuming to transport to the OR. For these reasons, the procedural suite was chosen as the site for the dual procedure.
Measurements were made of all our three procedural suites, and only one room was large enough to accommodate the lithotripter and portable c-arm. Outlets were installed in that room $(220 \mathrm{~V}$ for lithotripter and a data outlet for the c-arm monitor), and tape was placed on the floor indicating ideal placement of equipment (lithotripter, c-arm and c-arm monitor). PDSA cycles were performed to simulate the sequence of events that would occur once a patient was brought into the room. After several cycles, we determined that the lithotripter and fluoroscopy tables would start out side by side with a small gap. The patient would walk into the room and lie down, supine, on the fluoroscopy bed. The lithotripter would then be moved flush to the fluoroscopy table, and the awake patient would be asked to roll over to the prone position on the lithotripter. Next, positioning would be refined to ensure patient comfort and proper alignment of the lithotripter beam to the pancreatic stone. The patient would then roll back supine on the fluoroscopy table, and administration of anaesthesia would start. Once anaesthetised and intubated, the patient would be rolled back prone on the lithotripter for the pancreatic ESWL. On completion of lithotripsy, the patient would be rolled back supine on the fluoroscopy table for the ERCP. The lithotripter/c-arm/c-arm monitors would then all be removed from room to allow the gastroenterologist access to the patient for the ERCP. On completion of ERCP, the patient would be awakened from anaesthesia and transported to recovery. PDSA cycles were also conducted to simulate an emergency code situation to ensure a patient could be safely managed in the event of an arrhythmia during lithotripsy. 
Table 1 Patient characteristics, preintervetion and postintervention (1 August 2013) with intent-to-treat analysis

\begin{tabular}{lclll}
\hline & Total & Preintervention & Postintervention & Sig P \\
\hline Dates & & February 2011-July 2013 & August 2013-May 2017 \\
Number of patients & 292 & 119 & 173 & $55(14)$ \\
Age, mean (SD) & $54(15)$ & $53(15)$ & $87(50)$ & 0.27 \\
Sex, female (\%) & $152(52)$ & $65(55)$ & $1.8(3.6)$ & 0.47 \\
Hospital LOS, mean (SD) & $1.9(3.1)$ & $1.9(2.1)$ & $31(18)$ & 0.76 \\
Diabetes discharge, dx (\%) & $40(14)$ & $9(8)$ & $35(20)$ & 0.011 \\
Drug/ETOH abuse, dx (\%)† & $45(15)$ & $10(8)$ & $17(10)$ & 0.006 \\
Type, inpatient (\%) & $40(14)$ & $23(19)$ & 0.020 \\
\hline
\end{tabular}

${ }^{*} \mathrm{ICD} 9=250.00,250.02,250.81$ or ICD10=E11.9, E11.649, E11.65.

†ICD9=304, 305 or ICD10=F1X.

$\mathrm{ETOH}$, ethyl alcohol, ethanol, or alcohol; LOS, length of stay; Sig P, Significant $\mathrm{p}$ value.

\section{RESULTS}

Patient age and sex were similar before and after intervention, but more patients after the intervention had comorbidities of drug/alcohol abuse and diabetes. Despite this increase disease burden, hospital length of stay was unchanged (table 1).

Procedural characteristics are presented in table 2. A total of 292 cases were analysed from 2011 to 2017. Preintervention, $30 \%$ of the cases occurred on different days. Before the intervention, providers occasionally managed 'work arounds' to avoid two anaesthetics. Seventeen cases $(14 \%$ of total) were performed in rapid succession either in the lithotripsy OR (12 cases, ERCP completed using the low quality imaging of a portable c-arm) or by transporting the patients from the lithotripsy OR to the ERCP procedural suite while under general anaesthesia (five cases). Preintervention, the average delay between the ESWL and ERCP was over 8 hours. After the intervention, cases were done in immediate succession without exception. The total combined procedural time was unchanged, indicating that the work space was conducive to efficient procedural completion by both the urologists and gastroenterologists (figure 2). Procedure times were analysed for individual urologists and gastroenterologists, which also indicated no change in procedural times.

Anaesthesia charges are generated using a base charge plus a time component charge (15 min increments). So, although the procedural duration was unchanged, there was a reduction in the number of anaesthetics administered which eliminated a base charge. General anaesthesia with an endotracheal tube was the technique used preintervention for the ESWL. The anaesthesia technique preintervention for the ERCP varied between propofol anaesthesia without an endotracheal tube to general anaesthesia with an endotracheal tube. Postintervention, all procedures were done with general anaesthesia and an endotracheal tube. Based on billing charges, preintervention, on average, a patient would be charged for $87.5 \mathrm{~min}$ of anaesthesia during the pancreatic ESWL (CPT 50590, American Society of Anesthesiologists (ASA) code 00873, 5 based units +6 time units, $\$ 1078$ anaesthesia professional charge) plus $87.5 \mathrm{~min}$ for anaesthesia during the ERCP (CPT 43264, ASA code 000740, 5 based units+6 time units, \$1078 anaesthesia professional charge). Postintervention, the average billing charge would include $177 \mathrm{~min}$ of anaesthesia during the combined ESWL/ERCP procedure (CPT 43265, ASA code 00740, 5 based units+12 time units, \$1666 anaesthesia professional charge). Thus, billing charges generated for one patient undergoing the ESWL/ERCP procedure would be reduced $\$ 490$ by eliminating one anaesthetic base charge.

The topics of PSAs filed about the ESWL/ERCP procedure are listed in table 3. Those PSA topics that were specifically addressed during the intervention (aspirin

Table 2 ESWL/ERCP procedure details preintervention and postintervention

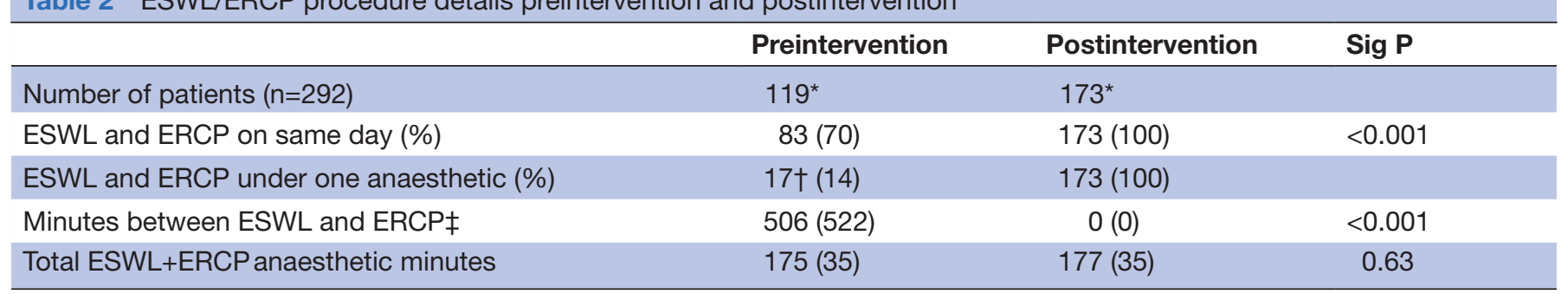

*Not all ERCP data are available; $n=100$ for minutes between procedures and for total minutes preintervention and $n=172$ postintervention. $\dagger 12$ cases the patient was transported under GA, five cases procedure was combined in lithotripsy OR. łERCP anaesthetic start - ESWL anaesthetic end in minutes; assume postintervention minutes $=0$.

ERCP, endoscopic retrograde cholangiopancreatography; ESWL, extracorporeal shock wave lithotripsy; OR, operating room. 


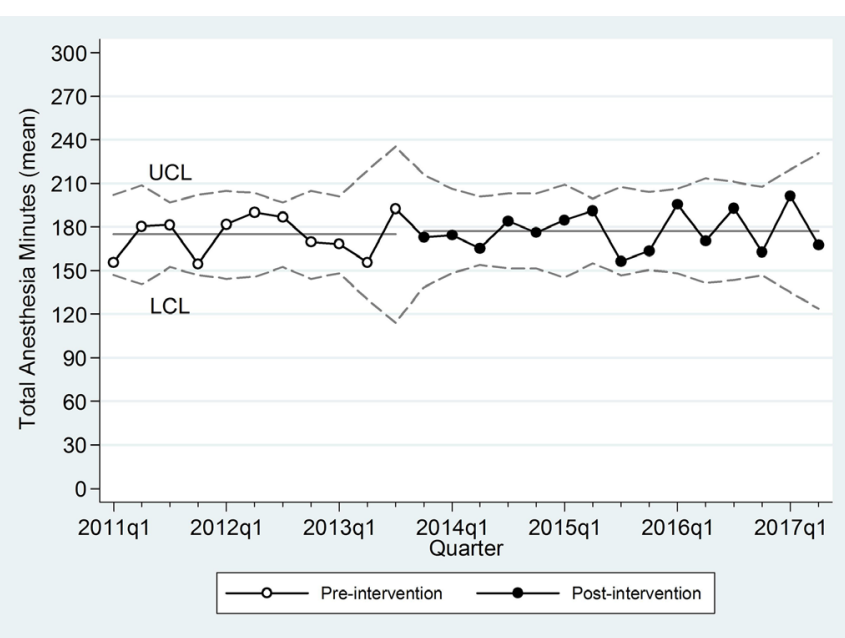

Figure 2 Statistical process control chart with total anaesthesia time (min) by quarter, upper confidence limit (UCL) and lower confidence limit (LCL).

ingestion, ERCP consent and two anaesthetics) were absent postintervention, though there remained the problem of two misplaced ESWL consents. Postintervention, PSAs related to the ESWL/ERCP procedure focused on clerical errors, non-standard scheduling (hospitalist scheduling for an inpatient) and inadequate workup, which were topics not addressed in the event. The lithotripter scheduling was addressed by a change in the categorisation of our lithotripter in our computer system to disallow double-booking of this equipment.

Lithotripsy repairs are graphically illustrated over time and indicate an increase in repairs immediately following the event, which could have been due to moving the lithotripter (figure 3). As the hospital has a maintenance contract for the lithotripter, these repairs did not incur

Table 3 Patient safety alert (PSA) topics before and after intervention

\section{Preintervention (2010-March 2013): 11 PSAs total}

\begin{tabular}{|c|c|}
\hline 4 & Cancellations due to ASA ingestion. \\
\hline 3 & Lack of ERCP consent delayed ESWL. \\
\hline 2 & $\begin{array}{l}\text { Cite patient safety issue of two anaesthetics when } \\
\text { should only be one. }\end{array}$ \\
\hline 2 & Lack of proper medical workup before procedure. \\
\hline \multicolumn{2}{|c|}{ Postintervention (May 2013-2017): 7 PSAs total } \\
\hline 2 & Lack of proper medical workup before procedure. \\
\hline 2 & $\begin{array}{l}\text { ESWL consent placed in wrong chart and urology } \\
\text { chart missing with ESWL consent. }\end{array}$ \\
\hline 1 & Gastroenterologist late delaying ESWL. \\
\hline 1 & $\begin{array}{l}\text { Inpatient scheduled by hospitalist with no } \\
\text { communication with Gl department. }\end{array}$ \\
\hline 1 & $\begin{array}{l}\text { Lithotripter table double booked due to scheduler } \\
\text { error, ESWL delay. }\end{array}$ \\
\hline
\end{tabular}

ERCP, endoscopic retrograde cholangiopancreatography; ESWL, extracorporeal shock wave lithotripsy.

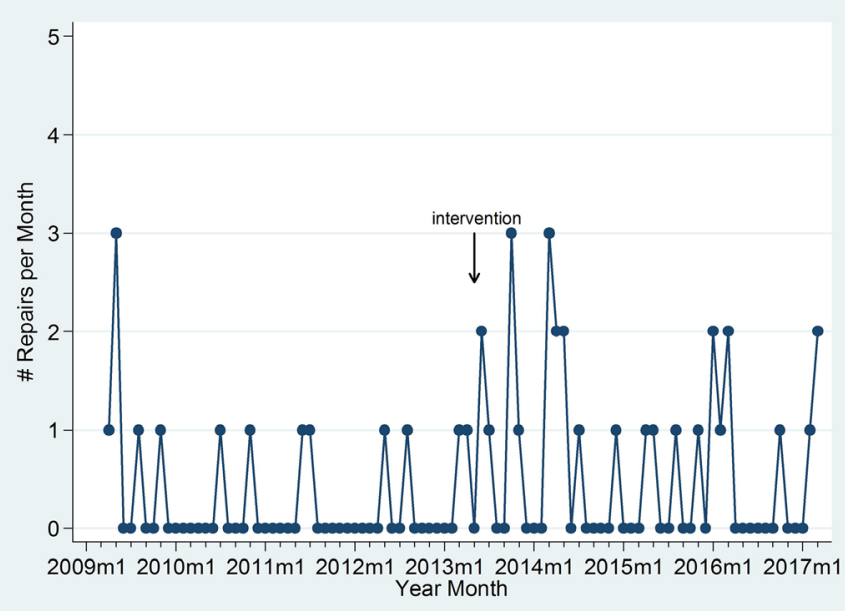

Figure 3 Lithotripter repairs per month before and after the intervention.

an additional charge to the hospital, although downtime for the unit may have impacted productivity.

Urologists were initially resistant to moving the lithotripter fearing it would impact productivity. This did not prove to be a problem, partly due to their serendipitous concurrent change in practice to treat ureteral stones by laser ureteroscopy rather than ESWL, which decreased competition for the lithotripsy unit. Our gastroenterologists were open to the change in practice, and the only concern regarding productivity stemmed from the early combined cases when the ESWL duration was less well established. Once the time to complete the ESWL was better understood, the gastroenterologists' schedules were adjusted to maximise efficiency.

\section{LESSONS AND LIMITATIONS}

Hospital staff seeing a problem such as the division of these two procedures and realising the obvious solution to combine them was not adequate to produce the magnitude of change required. An organised event with the support of hospital leadership was needed to bring about change. In this case, an important lesson was to focus on the patients' needs first, even if it required structural changes in the physical space. It is important to realise the quality of care was not compromised by the changes that led to this more patient-centred procedure line. Moving the lithotripter allowed the two procedures to be done in immediate succession with the resultant decrease in confusion, in-hospital travel and anaesthetics.

We addressed procedure delay by requiring both physicians to be present before the procedure. This approach is consistent with a GI endoscopy suite efficiency study showing the most common reason for delay was physician related (not present or incomplete paperwork) ${ }^{16}$ Despite the efforts, we still had PSAs written about delays after the intervention, one due lack of physician presence and the others due to clerical errors.

This work was specific to this one combined procedure and the ability to effect change hinged on the mobility 
of the lithotripter. However, the coordinated systematic approach to improving care may be applicable to other procedures. For example, a coordinated procedures has emerged where a gastroenterologists and an interventional radiologist team up to provide 'dual drainage' of walled off pancreatic necrosis. This procedure also requires anaesthesia and therefore the coordination of three services.

To ascertain success in this Lean project metrics of interest include improved patient access, experience and safety. However, measurements in real time were challenging. We attempted to quantify patient access by reviewing charts to determine the time lag from first contact with a patient to date of procedure. Unfortunately, after reviewing several charts, it was apparent that the first phone contact was not reliably captured in our electronic medical records. We also explored first gastroenterologist contact to date of procedure but, unfortunately, many of these cases occur with out-of-town patients who met the gastroenterologist for the first time on the day of procedure. We also wanted to capture improvement in patient experience, but historically there are no ESWL/ERCP specific questionnaires and when we cross-referenced our general hospital questionnaire responses against our ESWL/ERCP patient list, we did not find any matches.

If we were to do this project again, we would look for a way to quantify the scheduling efficiency. This could be done by setting up a daily query where scheduling accuracy and call metrics (time to answer call and duration of call) could be collected in order to quantify improvements in scheduling. ${ }^{15}$ There may have been value in using the hard modelling technique of discreet event simulation to track and simulate the patient journey in order quantify the impact of our intervention. ${ }^{17}$ Collecting patient outcome and satisfaction data would also have been valuable, and a research project collecting that data would strengthen the conclusion that this process improvement positively impacted patient care.

\section{CONCLUSION}

The combination of ESWL/ERCP allows treatment of large pancreatic stones in 1 day that otherwise would require surgery. We were able to half the number of anaesthetics for each patient, thereby reducing the time spent in the most dangerous phases of anaesthesia, namely induction and emergence.

By rethinking the entire process from scheduling through postprocedure recovery focusing on the patient's perspective, we were able to make this process safer and more efficient.

Acknowledgements The RPIW team members: Michael Glenn, MD; Shelly Powell; Valerie Marquardt; Paul Kozlowski, MD; Tracey Jones, RN; Jessica Roman; Ruth Sonsteng; Patricia DesSayles; Jennifer Bradley; Dianna Davis; and Aaron Villarreal, MD (authors WW and AR were also team members).
Contributors WAW contributed to the planning, conduct and reporting of the work described in the article and is responsible for the overall content as guarantor. $M G$ and OSL contributed to the planning and reporting of the work described in the article. ASR contributed to the planning and conduct of the work described in the article. BLW and CCB contributed to the reporting of the work described in the article. All authors provided editorial input and approved the final document for publication.

Funding The authors have not declared a specific grant for this research from any funding agency in the public, commercial or not-for-profit sectors.

Competing interests None declared.

Patient consent Not required.

Provenance and peer review Not commissioned; externally peer reviewed.

Open access This is an open access article distributed in accordance with the Creative Commons Attribution Non Commercial (CC BY-NC 4.0) license, which permits others to distribute, remix, adapt, build upon this work non-commercially, and license their derivative works on different terms, provided the original work is properly cited, appropriate credit is given, any changes made indicated, and the use is non-commercial. See: http://creativecommons.org/licenses/by-nc/4.0/.

\section{REFERENCES}

1. Seven G, Schreiner MA, Ross AS, et al. Long-term outcomes associated with pancreatic extracorporeal shock wave lithotripsy for chronic calcific pancreatitis. Gastrointest Endosc 2012;75:997-1004.

2. Ross AS, Gluck M. Sa1451 Systematic process improvement applied to scheduling and performing ESWL followed by ERCP for large pancreatic duct calculi: waste reduction and quality improvement. Gastrointest Endosc 2014;79:AB217.

3. Kenney C. Transforming health care: Virginia Mason Medical Center's pursuit of the perfect patient experience. New York, NY: Productivity Press-Taylor \& Francis Group, 2011.

4. Kozarek R. Recent research on extracorporeal shock wave lithotripsy with ERCP for treatment of chronic calcific pancreatitis. Gastroenterol Hepatol 2012;8:478-81.

5. Kozarek RA, Brandabur JJ, Ball TJ, et al. Clinical outcomes in patients who undergo extracorporeal shock wave lithotripsy for chronic calcific pancreatitis. Gastrointest Endosc 2002;56:496-500.

6. Korpela T, Udd M, Tenca A, et al. Long-term results of combined ESWL and ERCP treatment of chronic calcific pancreatitis. Scand J Gastroenterol 2016;51:866-71.

7. Bhandari S, Maydeo A. Extracorporeal shock wave lithotripsy: the cornerstone of pancreatic endotherapy. Gastrointest Endosc 2016;84:79-80.

8. Tandan M, Talukdar R, Reddy DN. Management of pancreatic calculi: an update. Gut Liver 2016;10:873-80.

9. Guda NM, Partington S, Freeman ML. Extracorporeal shock wave lithotripsy in the management of chronic calcific pancreatitis: a metaanalysis. JOP 2005;6:6-12.

10. Milovic V, Wehrmann T, Dietrich CF, et al. Extracorporeal shock wave lithotripsy with a transportable mini-lithotripter and subsequent endoscopic treatment improves clinical outcome in obstructive calcific chronic pancreatitis. Gastrointest Endosc 2011;74:1294-9.

11. Del Río AS, Baudet JS, Fernández OA, et al. Evaluation of patient satisfaction in gastrointestinal endoscopy. Eur J Gastroenterol Hepatol 2007;19:896-900.

12. Sewitch MJ, Gong S, Dube C, et al. A literature review of quality in lower gastrointestinal endoscopy from the patient perspective. Can J Gastroenterol 2011;25:681-5.

13. Hydes T, Hansi N, Trebble TM. Lean thinking transformation of the unsedated upper gastrointestinal endoscopy pathway improves efficiency and is associated with high levels of patient satisfaction. BMJ Qual Saf 2012;21:63-9.

14. Kaplan GS, Patterson SH, Ching JM, et al. Why Lean doesn't work for everyone. BMJ Qual Saf 2014;23:970-3.

15. Fairchild EM, Lambert KL, Talley MI, et al. Cross-site scheduling of endoscopic procedures improves efficiency while maintaining patient safety. J Healthc Qual 2016;38:24-33.

16. Yong E, Zenkova O, Saibil F, et al. Efficiency of an endoscopy suite in a teaching hospital: delays, prolonged procedures, and hospital waiting times. Gastrointest Endosc 2006;64:760-4.

17. Pitt M, Monks T, Crowe S, et al. Systems modelling and simulation in health service design, delivery and decision making. BMJ Qual Saf 2016;25:38-45. 